\title{
Optimization of Power Consumption in VLSI Circuit
}

\author{
Mr. Praveen Kumar Gupta ${ }^{1}$, Jagdeep Kaliraman ${ }^{2}$ \\ ${ }^{I}$ Associate Professor CSE Deptt. LU (Faridabad) \\ ${ }^{2}$ A P, ECE Deptt. ACEM (Faridabad)
}

\begin{abstract}
Increasing speed and complexity of design gives a significant increase in power consumption in VLSI chips. Speed, power consumption and space are major issues in VLSI circuit. To meet these challenges there are certain design techniques which are used to reduce power. Optimization of power can be done by considering various components such as transistor sizing, voltage scaling, variable $V_{D D}$, multiple threshold voltages, voltage islands, power gating, long channel transistor, stacking \& parking states and logic styles etc. Power consumption in VLSI circuit is data dependent. In this paper different design methods are tested to optimize the power. It is found that algorithm based design reduces gate switching activity that results reduction of power in multiplier circuit.
\end{abstract}

Keyword: Transistor sizing, voltage scaling, variable $V_{D D}$, multiple threshold voltages, voltage islands, long channel transistor, stacking \& parking states, logic styles, Genetic algorithm, Booth multiplication

\section{Introduction}

Reduction in power dissipation is an important design issue in VLSI circuits. The design parameters have major effect on the overall performance of the system. On the basis of component used and their function different optimization techniques can be used. For example in case of multiplier power consumption depends upon data. This is because switching contributes to more power consumption. This can be optimized by using various gate combinations. Gate switching can be reduced by using algoritms. For example of in case of multiplier design method Booth algorithm as well as modified Booth algorithm can be used for efficient multiplication. In this paper various approaches are used for power consumption eg. transistor sizing, voltage scaling, variable $\mathrm{V}_{\mathrm{DD}}$, multiple threshold voltages, voltage islands, long channel transistor, stacking\& parking states, logic styles, Genetic algorithm, Booth multiplication etc.

This paper is divided into five sections. First section is of introduction. Second section is about preliminary studies. Third section includes different design methods and their testing. Fourth section is of comparison with discussion. Finally, fifth section is conclusion of the work reported here.

\subsection{Power Dissipation}

\section{Preliminaries}

In case of VLSI circuits power dissipation is of two types' i.e. static power dissipation and dynamic power dissipation. This is shown as under:-

1. Static Power Dissipation

2. Dynamic Power Dissipiation

Static power dissipation is due to three major factors as leakage current, sub threshold current of transistors and tunnelling effect of current. So optimization can be achieved by concentrating all these design issues. As we know that MOS transistor has two P-N junctions: one is between source and drain and other is between drain and source. When source substrate junction or drain substrate junction is reversed biased. The leakage current flows through reverse biased P-N junctions. Generally this leakage current is very small in magnitude so we ignore this current.

Leakage current is temperature dependent. This is because leakage current is caused by thermally generated carriers. With increase in temperature, leakage current increases. According to a survey it is found that it becomes double for every $10^{\circ} \mathrm{C}$ rise in temperature. From this it is clear that temperature of a circuit must be controlled at any cost.

\subsection{Sub threshold current of transistors}

At VGS $=\mathrm{V}_{\mathrm{T}}$ surface is said to be inverted. In this condition there is strong inversion and conduction takes place afterwards. But practically $\mathrm{V}_{\mathrm{GS}}<\mathrm{V}_{\mathrm{T}}$. MOS transistor is under sub threshold or weak inversion conduction. This current is equally small in magnitude and rises exponentially. This current flows from drain to source. It is observed at $\mathrm{V}_{\mathrm{GS}}=0$. 
If $\mathrm{V}_{\mathrm{T}}$ is nearly zero volt. Then threshold leakage current will be more. From this it is clear that if threshold voltage is less leakage current will be more. Hence (PD) static will also be more. The threshold voltage has to keep high.

As $\left(\mathrm{P}_{\mathrm{D}}\right)_{\text {static }}=\mathrm{P}(\mathrm{t})=\mathrm{i}_{\mathrm{DD}}(\mathrm{t}) \mathrm{V}_{\mathrm{DD}}$

Here $i_{D D}(t)$ is the leakage current or static current and $V_{D D}$ is supply voltage. From this it is clear that if leakage current increases, then static power increases. Hence threshold voltage represents a trade off between performance and static power dissipiation.

\subsection{Tunnelling Current Effect}

As gate oxide material is $\mathrm{SiO}_{2}$ and $\mathrm{SiO}_{2}$ is very good dielectric. The leakage current through this good dielectric is very low. But if the thickness of dielectric is very less. Then electrons can tunnel through this dielectric. If the thickness of dielectric decreases tunnelling effect increases.

\subsection{Dynamic Power Dissipiation}

Dynamic power dissipiation occurs in charging and discharging of capacitive loads. Let $\mathrm{C}_{\mathrm{L}}$ is the capacitive load. If this capacitive load is switched between ground and $\mathrm{V}_{\mathrm{DD}}$ and switching frequency is taken as

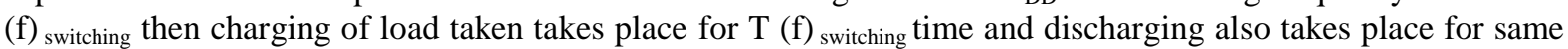
time, $\mathrm{T}$ being the time interval.

During charging current flows from $V_{D D}$ to $C_{L}$ and during discharging current flows from $C_{L}$ to $V_{D D}$. Total charge transferred from positive supply rail $\left(\mathrm{V}_{\mathrm{DD}}\right)$ to negative supply rail (ground) during charging and discharging cycle will be:-

$\mathrm{Q}=\mathrm{C}_{\mathrm{L}} \mathrm{V}_{\mathrm{DD}}$

So $\left(\mathrm{P}_{\mathrm{D}}\right)_{\text {Dynamic }}=\frac{1}{T} \int_{0}^{\mathrm{T}} \mathrm{i}_{\mathrm{DD}}(\mathrm{t}) \mathrm{V}_{\mathrm{DD}} \mathrm{dt}=\frac{V D D}{T} \int_{0}^{\mathrm{T}} \mathrm{i}_{\mathrm{DD}}(\mathrm{t}) \mathrm{dt}$

$=\frac{1}{T} \mathrm{~V}_{\mathrm{DD}}\left[\mathrm{T}(\mathrm{f})_{\text {switching }} \mathrm{C}_{\mathrm{L}} \mathrm{V}_{\mathrm{DD}}\right]$

$=\mathrm{C}_{\mathrm{L}} V_{D D}^{2}(\mathrm{f})_{\text {switching }}$

$\left(\mathrm{P}_{\mathrm{D}}\right)_{\text {Total }}=(\mathrm{PD})_{\text {switching }}+\left(\mathrm{P}_{\mathrm{D}}\right)_{\text {dynamic }}$

Usually (PD) Total can be ignored but high sub threshold current if exist, then it cannot be ignored. But $\left(\mathrm{P}_{\mathrm{D}}\right)_{\text {dynamic }}$ is far greater than (PD) $)_{\text {static }}$ hence it cannot be ignored.

At this short span of time, rail to rail current flows and causes power dissipiation which can not be avoided.

Table 1

\begin{tabular}{|l|}
\hline Sources to bear power dissipiation \\
\hline Air cooled system \\
Heat sinks \\
Liquid cooling \\
\hline
\end{tabular}

Dynamic power dissipiation affects the battery when the device is in active mode but static power battery life even when the device is in sleep mode.

\section{Power Dissipation Estimation}

In all logic circuits power consumption is related to information transfer and each circuit have inherent requirement of information transfer. Let $\mathrm{R}$ is transfer rate requirement for a given architecture. The lower bound of this rate may be determined which can be used for power dissipiation estimation. The different architecture can perform same function but may have different information transfer rate and channel capacity. The channel capacity can be given as under:-

$$
c=\int_{0}^{W} \operatorname{los}_{2}[1+\operatorname{sNR}(f)] d f
$$

Here SNR stands for signal to noise ratio. For any transfer, capacity should be greater than or equal to $\mathrm{R}$. The overall power in digital circuit is a function of signal power, temperature and semiconducting property etc. However power dissipiation is mainly due to ground bounce. The lower bound of power dissipiation can be calculated using information transfer capacity of channel. Let $\mathrm{R}$ be the required information transfer rate, $\mathrm{W}$ is the channel bandwidth \& $\mathrm{C}$ is the channel capacity. Lower bound of power dissipiation can be given as:-

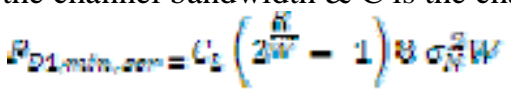

\subsection{Power Optimization as Data dependent}

\section{Design methods for optimisation}

Complexity of data contributes to switching activity in the circuit. With the use of efficient algorithm design component of circuit can be reduced. By the application of simulation to standard design and comparing 
it with optimization better design component can be found. Gate switching for all initial states and all inputs can be simulated to analyse power consumption. Data dependency is helpful in gate design complexity. Ordering of gate inputs affect both power and delay. Prasad [1] has described methods to optimize the power and/or delay of logic gates based on transistor reordering. So, considerable improvements in power and delay can be obtained by proper ordering of transistors. For instance, late arriving signals can be placed closer to the output to minimize gate propagation delay. Another approach to reduce power is to consider the size of gate, which has significant impact on circuit delay and power dissipation. By increasing the size of transistors in a given gate, delay of the gate can be decreased but in contrast, power dissipated in the gate and fabrication space increases. Therefore, an optimum balance can be achieved by sizing of transistors appropriately. A method is to compute the slack at each gate in the circuit, where the slack of a gate corresponds to how much the gate can be slowed down without affecting the critical delay of the circuit. Alternatively, in different sub - circuits, where slack is greater than zero are utilized and the size of the transistors is reduced until the slack becomes zero, or the transistors attain a minimum size.

\section{Combinational Gate Level Design}

In gate level design of circuit, different combination of logical gates may different combination of logical gates may produce same circuit output but different value of power consumption. Path balancing, factorization and don't care optimization may be utilized to optimize power consumption. Path balancing can be achieved by avoiding delay at each input gate. Genetic Algorithm can be used to determine different combination of gates and power consumption can be formulated by devising Fitness Function. Coello [3] has proposed design of combinational logic circuit based on Genetic Algorithm.

By defining chromosome development schemes of various combinations of logic circuit can be developed using cross over and mutation. This approach is more efficient than human designer as various constraint of design circuit can be devised subject to fitness function. Genetic Algorithm can reduce number of gates, which consequently reduce power consumption; as the work of Coello [2], shows on 2-bit adder and 2-bit multiplier with a particular 'cardinality' that $56 \%$ reduction in number of gates for the circuit can be achieved.

\section{Comparison and discussion}

Number of Gates versus power saving in CMOS - based on ISCAS-89 benchmark circuits. [8]

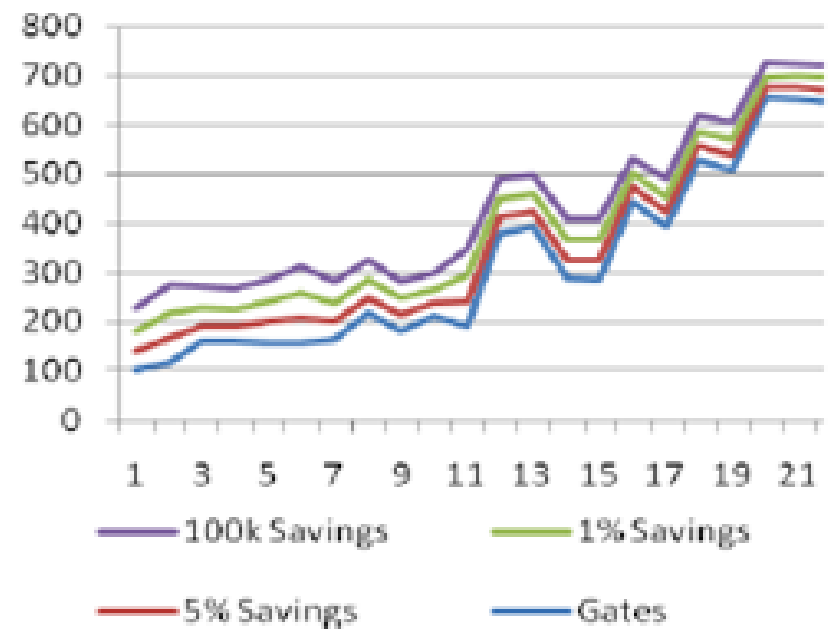

(Graph-1) Gate V's Power based on the work of Jonathan P. Halter and Farid N. Najm [8]

Table 2. Power reduction versus Speed Loss 


\begin{tabular}{|c|c|c|}
\hline $\begin{array}{c}\text { Power } \\
\text { Reduction in } \\
\text { supply voltage }\end{array}$ & $\begin{array}{l}\text { Speed } \\
\text { Loss }\end{array}$ & $\begin{array}{l}\text { Constraints/ } \\
\text { Specifications }\end{array}$ \\
\hline $\begin{array}{c}\text { leakage } \\
\text { power reductions } \\
\text { up to } 54 \%\end{array}$ & $\begin{array}{l}\text { Not } \\
\text { reported }\end{array}$ & $\begin{array}{l}\text { "Logic design to } \\
\text { reduce } \\
\text { the leakage power of } \\
\text { CMOS circuits that } \\
\text { use clock gating } \\
\text { to reduce the } \\
\text { dynamic power } \\
\text { dissipation tested on } \\
\text { ISCAS-89 } \\
\text { benchmark circuits" } \\
\text { [8] }\end{array}$ \\
\hline $\begin{array}{c}0.13 \mathrm{~V} \\
\text { or } \\
800 \text { times } \\
\end{array}$ & 19 times & $\begin{array}{l}\text { "0.5-fim gate length } \\
\text { and static logic" [9] }\end{array}$ \\
\hline $\begin{array}{l}\text { "1.1 V supply and } \\
\text { consumes less than } \\
5 \text { mW-which is } \\
\text { more than three } \\
\text { orders of } \\
\text { magnitude lower } \\
\text { powver compared to } \\
\text { equivalent } \\
\text { commercial } \\
\text { solutions." [10] }\end{array}$ & $\begin{array}{l}\text { Not } \\
\text { reported }\end{array}$ & - \\
\hline
\end{tabular}

For instance, a modular approach as shown below in Fig. $\mathbf{1}$ is proposed to adopt appropriate optimization technique considering different possibilities in multiplier design.

Figure1. Modular approach for multiplier design

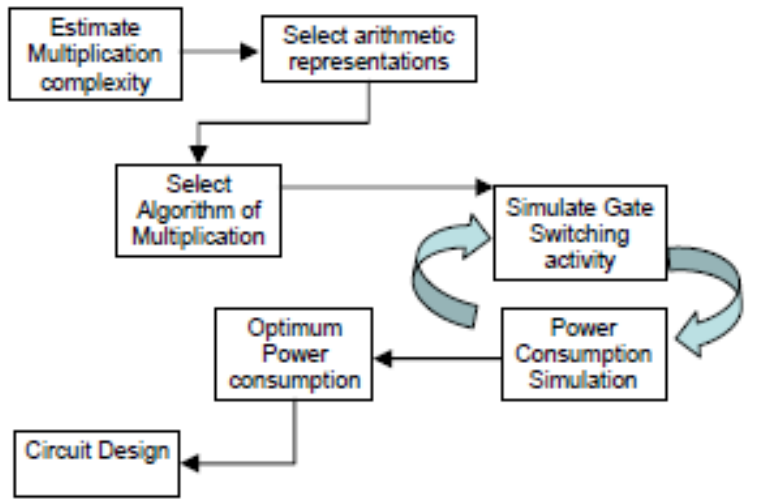

\section{Conclusion}

It is found that data complexity and various combination of gate level digital circuit has considerable impact in power dissipiation. Besides, this physical design can be optimized by using Genetic algorithm by analysing the placement option. This is subjected to optimum space allocation. Similarly selection of Booth algorithm may reduce power consumption of data complexity. It is found that in multiplier circuit Modified Booth Algorithm reduces power consumption as compared to other methods of multiplication.

\section{References}

[1] S. C. Prasad and K. Roy, "Circuit Optimization for Minimization of Power Consumption Under Delay Constraint". In Proceedings of the Int'l Workshop on Low Power Design, pages 15-20, April 1994.

[2] Coello, Carlos A., Christiansen, Alan D. \& Hernández Aguirre, "Using Genetic Algorithms to Design Combinational Logic Circuits." ANNIE'96. Intelligent Engineering through Artificial Neural Networks, Volume 6. Smart Engineering Systems: Neural Networks, Fuzzy Logic and Evolutionary Programming. Edited by Cihan H. Dagli, Metin Akay, C. L. Philip Chen, Benito R. Fernandez and Joydeep Ghosh. pp 391-396. November, 1996.

[3] Coello, Carlos Artemio Coello. "An Empirical Study of Evolutionary Techniques for Multi-objective Optimization in Engineering Design”. Ph.D. Dissertation, Department of Computer Science, Tulane University, New Orleans, LA, 1996.

[4] H. J. M. Veendrick, "Short-circuit dissipation of static CMOS circuit and its impact on the design of buffer circuits," IEEE J. SolidState Circuits, Vol. 19, Aug 1984, pp. 468-473.

[5] Charles H. Bennett, "Notes on Landauer's Principle, Reversible Computation and Maxwell's Demon", Studies in History and Philosophy of Modern Physics, v. 34, pp. 501-510, 2003

[6] C.H. Bennett, "The Thermodynamics of Computation - a Review” Internat. J. Theoret. Phys. 21, pp. 905-940 (1982).

[7] L. Benini, M. Favalli, and B. Ricco, "Analysis of hazard contribution to power dissipation in CMOS IC's". In Proceedings of the 1994 International Workshop on Low Power Design, pp 27-32, April 1994. 
[8] Vaughn Betz and Jonathan Rose, "Circuit Design, Transistor Sizing and Wire Layout of FPGA Interconnect", IEEE Custom Integrated Circuits Conference, 1999

[9] Boemo, E., Gonzalez de Rivera, G., Lopez-Buedo, S., Meneses, J., "Some R.W. Allen. Addison-Wesley, 1996.

[10] Alain Guyot and Sélim Abou-Samra, "Low Power CMOS Digital Design", In proc. Of International Conference on Microelectronics 1998 (ICM’98), Monastir, Tunisia, December 1998

[11] R. Landauer, "Irreversibility and Heat Generation in the Computing Process", IBM Journal of Research and Development, Vol 5, pp. 261-269, 1961

[12] P. Landman, Low-Power Architectural Design Methodologies, Ph. D. Thesis, Electronic Research Laboratory, university of California, Berkeley, August 1994

[13] Fei Li, Deming Chen, Lei He, Jason Cong: “Architecture evaluation for power efficient FPGAs”, Proc. Of Int. Symp on Field Programmable Gate Arrays, 2003, pp. 175-184

[14] F. N. Najm and M. G. Xakellis, "Statistical estimation of the switching activity in VLSI circuits", VLSI Design, vol. 7, no. 3, pp. 243-254, 1998

[15] M. Pedram, "Power estimation and optimization at the logic level," Int'l Journal of High Speed Electronics and Systems, Vol. 5, No. 2 (1994), pp. 179-202.

[16] Andrés David García García, "Etude sur l'Estimation et l'Optimisation de la Consommation de puissance”, PhD Thesis, l'Ecole Nationale Supérieure des Télécommunications, Paris, 2000.

[17] Alain Guyot and Sélim Abou-Samra, "Low Power CMOS Digital Design", In proc. Of International Conference on Microelectronics 1998 (ICM'98), Monastir, Tunisia, December 1998.

[18] P. Schneider and S. Krishnamoorthy. "Effects of correlations on accuracy of power analysis - an experimental study", International Symposium on Low Power Electronics and Design, Monterey, California, United States, 1996, pp. 113-116.

[19] Jan M. Rabaey and Massoud Pedram. "Low power design methodologies". Boston, Kluwer Academic, 1996.

[20] Kara K.W. Poon, Steven J.E. Wilton, and A. Yan, "A Detailed Power Model for Field-Programmable Gate Arrays," ACM Transactions on Design Automation of Electronic Systems (TODAES), vol. 10, issue 2, pp. 279-302, April 2005.

[21] M. Pedram, "Design technologies for Low Power VLSI", In Encyclopedia of Computer Science and Technology, Vo. 36, Marcel Dekker, Inc., 1997, pp. 73-96.

[22] F. N. Najm and M. G. Xakellis, "Statistical estimation of the switching activity in VLSI circuits", VLSI Design, vol. 7, no. 3, pp. 243-254, 1998

[23] Fei Li, Deming Chen, Lei He, Jason Cong: "Architecture evaluation for power efficient FPGAs", Proc. Of Int. Symp on Field Programmable Gate Arrays, 2003, pp. 175-184

[24] P. Landman, Low-Power Architectural Design Methodologies, Ph. D. Thesis, Electronic Research Laboratory, University of California, Berkeley, 1994

[25] R. Landauer, "Irreversibility and Heat Generation in the Computing Process", IBM Journal of Research and Development, Vol 5, N 3, pp. 261-269, 1961.

[26] James Kao, Siva Narendra, Anantha Chandrakasan, "Sub threshold leakage modeling and reduction techniques", In proc. of the 2002 IEEE/ACM international conference on Computer-Aided Design, pp. 141-148, 2002

[27] Vaughn Betz and Jonathan Rose, "Circuit Design, Transistor Sizing and Wire Layout of FPGA Interconnect", IEEE Custom Integrated Circuits Conf., 1999.

[28] Charles H. Bennett, "Notes on Landauer's Principle, Reversible Computation and Maxwell's Demon", Studies in History and Philosophy of Modern Physics, v. 34, pp. 501-510, 2003.

[29] Boemo, E., Gonzalez de Rivera, G., Lopez-Buedo, S., Meneses, J., "Some Notes on Power Management on FPGAs", LNCS, No. 975, Springer-Verlag, Berlin, pp 149-157, 1995. 